Article

\title{
The Randomized First-Hitting Problem of Continuously Time-Changed Brownian Motion
}

\author{
Mario Abundo \\ Dipartimento di Matematica, Università Tor Vergata, 00133 Rome, Italy; abundo@mat.uniroma2.it; \\ Tel.: +390672594627
}

Received: 4 April 2018; Accepted: 25 May 2018; Published: 28 May 2018

check for updates

\begin{abstract}
Let $X(t)$ be a continuously time-changed Brownian motion starting from a random position $\eta, S(t)$ a given continuous, increasing boundary, with $S(0) \geq 0, P(\eta \geq S(0))=1$, and $F$ an assigned distribution function. We study the inverse first-passage time problem for $X(t)$, which consists in finding the distribution of $\eta$ such that the first-passage time of $X(t)$ below $S(t)$ has distribution $F$, generalizing the results, valid in the case when $S(t)$ is a straight line. Some explicit examples are reported.
\end{abstract}

Keywords: first-passage time; inverse first-passage problem; diffusion

\section{Introduction}

This brief note is a continuation of [1,2]. Let $\sigma(t)$ be a regular enough non random function, and let $X(t)=\eta+\int_{0}^{t} \sigma(s) d B_{s}$, where $B_{t}$ is standard Brownian motion (BM) and the initial position $\eta$ is a random variable, independent of $B_{t}$. Suppose that the quadratic variation $\rho(t)=\int_{0}^{t} \sigma^{2}(s) d s$ is increasing and $\rho(+\infty)=\infty$, then there exists a standard BM $\widetilde{B}$ such that $X(t)=\eta+\widetilde{B}(\rho(t))$, namely $X(t)$ is a continuously time-changed BM (see e.g., [3]). For a continuous, increasing boundary $S(t)$, such that $P(\eta \geq S(0))=1$, let

$$
\tau=\tau_{S}=\inf \{t>0: X(t) \leq S(t)\}
$$

be the first-passage time (FPT) of $X(t)$ below $S$. We assume that $\tau$ is finite with probability one and that it possesses a density $f(t)=\frac{d F(t)}{d t}$, where $F(t)=P(\tau \leq t)$. Actually, the FPT of continuously time-changed BM is a well studied problem for constant or linear boundary and a non-random initial value (see e.g., [4-6]).

Assuming that $S(t)$ is increasing, and $F(t)$ is a continuous distribution function, we study the following inverse first-passage-time (IFPT) problem:

given a distribution $F$, find the density $g$ of $\eta$ (if it exists) for which it results $P(\tau \leq t)=F(t)$.

The function $g$ is called a solution to the IFPT problem. This problem, also known as the generalized Shiryaev problem, was studied in $[1,2,7,8]$, essentially in the case when $X(t)$ is BM and $S(t)$ is a straight line; note that the question of the existence of the solution is not a trivial matter (see e.g., [2,7]). In this paper, by using the properties of the exponential martingale, we extend the results to more general boundaries $S$.

The IFPT problem has interesting applications in mathematical finance, in particular in credit risk modeling, where the FPT represents a default event of an obligor (see [7]) and in diffusion models for neural activity ([9]). 
Notice, however, that another type of inverse first-passage problem can be considered: it consists in determining the boundary shape $S$, when the FPT distribution $F$ and the starting point $\eta$ are assigned (see e.g., [10-13]).

The paper is organized as follows: Section 2 contains the main results, in Section 3 some explicit examples are reported; Section 4 is devoted to conclusions and final remarks.

\section{Main Results}

The following holds:

Theorem 1. Let be $S(t)$ a continuous, increasing boundary with $S(0) \geq 0, \sigma(t)$ a bounded, non random continuous function of $t>0$, and let $X(t)=\eta+\int_{0}^{t} \sigma(s) d B_{s}$ be the integral process starting from the random position $\eta \geq S(0)$; we assume that $\rho(t)=\int_{0}^{t} \sigma^{2}(s) d s$ is increasing and satisfies $\rho(+\infty)=+\infty$. Let $F$ be the probability distribution of the FPT $\tau_{S}$ of X below the boundary $S$ ( $\tau_{S}$ is a.s. finite by virtue of Remark 3 ). We suppose that the r.v. $\eta$ admits a density $g(x)$; for $\theta>0$, we denote by $\widehat{g}(\theta)=E\left(e^{-\theta \eta}\right)$ the Laplace transform of $g$.

Then, if there exists a solution to the IFPT problem for $X$, the following relation holds:

$$
\widehat{g}(\theta)=\int_{0}^{+\infty} e^{-\theta S(t)-\frac{\theta^{2}}{2} \rho(t)} d F(t)
$$

Proof. The process $X(t)$ is a martingale, we denote by $\mathcal{F}_{t}$ its natural filtration. Thanking to the hypothesis, by using the Dambis, Dubins-Schwarz theorem (see e.g., [3]), it follows that the process $\widetilde{B}(t)=X\left(\rho^{-1}(t)\right)$ is a Brownian motion with respect to the filtration $\mathcal{F}_{\rho^{-1}(t)}$; so the process $X(t)$ can be written as $X(t)=\eta+\widetilde{B}(\rho(t))$ and the FPT $\tau$ can be written as $\tau=\inf \{t>0: \eta+\widetilde{B}(\rho(t)) \leq S(t)\}$. For $\theta>0$, let us consider the process $Z_{t}=e^{-\theta X(t)-\frac{1}{2} \theta^{2} \rho(t)}$; as easily seen, $Z_{t}$ is a positive martingale; indeed, it can be represented as $Z_{t}=e^{-\theta X(0)}-\theta \int_{0}^{t} Z_{s} \sigma(s) d B_{s}$ (see e.g., Theorem 5.2 of [14]). We observe that, for $t \leq \tau$ the martingale $Z_{t}$ is bounded, because $X(t)$ is non negative and therefore $0<Z_{t} \leq e^{-\theta X(t)} \leq 1$. Then, by using the fact that, for any finite stopping time $\tau$ one has $E\left[Z_{0}\right]=E\left[Z_{\tau \wedge t}\right]$ (see e.g., Formula (7.7) in [14]), and the dominated convergence theorem, we obtain that

$$
\begin{gathered}
E\left[Z_{0}\right]=E\left[e^{-\theta X(0)}\right]=E\left[e^{-\theta \eta}\right]=\lim _{t \rightarrow \infty} E\left[e^{-\theta X(\tau \wedge t)-\frac{1}{2} \theta^{2} \rho(\tau \wedge t)}\right] \\
=E\left[\lim _{t \rightarrow \infty} e^{-\theta X(\tau \wedge t)-\frac{1}{2} \theta^{2} \rho(\tau \wedge t)}\right]=E\left[e^{-\theta S(\tau)-\frac{1}{2} \theta^{2} \rho(\tau)}\right] .
\end{gathered}
$$

Thus, if $\widehat{g}(\theta)=E\left(e^{-\theta \eta}\right)$ is the Laplace transform of the density of the initial position $\eta$, we finally get

$$
\widehat{g}(\theta)=E\left[e^{-\theta S(\tau)-\frac{\theta^{2}}{2} \rho(\tau)}\right]
$$

that is Equation (2).

Remark 1. If one takes in place of $X(t)$ a process of the form $\widetilde{X}(t)=\eta S(t)+S(t) B(\rho(t))$, with $\eta \geq 1$, that is, a special case of continuous Gauss-Markov process ([15]) with mean $\eta S(t)$, then $\widetilde{X}(t) / S(t)$ is still a continuously time-changed BM, and so the IFPT problem for $\widetilde{X}(t)$ and $S(t)$ is reduced to that of continuously time-changed BM and a constant barrier, for which results are available (see e.g., [4-6]).

Remark 2. By using Laplace transform inversion (when it is possible), Equation (4) allows to find the solution $g$ to the IFPT problem for $X$, the continuous increasing boundary $S$, and the distribution $F$ of the FPT $\tau$. Indeed, some care has to be used to exclude that the found distribution of $\eta$ has atoms together with a density. However, as already noted in [2,7], the function $\widehat{g}$ may not be the Laplace transform of some probability density function, so in that case the IFPT problem has no solution; really, it may admit more than one solution, since the right-hand member of Equation (4) essentially furnishes the moments of $\eta$ of any order $n$, but this is not always sufficient 
to uniquely determine the density $g$ of $\eta$. In line of principle, the right-hand member of Equation (4) can be expressed in terms of the Laplace transform of $f(t)=F^{\prime}(t)$, though it is not always possible to do this explicitly. A simple case is when $S(t)=a+b t$, with $a, b \geq 0$, and $\rho(t)=t$, that is, $X(t)=B_{t}(\sigma(t)=1)$; in fact, one obtains

$$
\widehat{g}(\theta)=E\left[e^{-\theta(a+b \tau)-\frac{\theta^{2}}{2} \tau}\right]=e^{-\theta a} E\left[e^{-\theta\left(b+\frac{\theta}{2}\right) \tau}\right]=e^{-\theta a} \widehat{f}\left(\frac{\theta(\theta+2 b)}{2}\right),
$$

which coincides with Equation (2.2) of [2], and it provides a relation between the Laplace transform of the density of the initial position $\eta$ and the Laplace transform of the density of the FPT $\tau$.

Remark 3. Let $S(t)$ be increasing and $S(0) \geq 0$, then $\tau$ is a.s. finite; in fact $\tilde{\tau}=\rho(\tau)=\inf \{t>0$ : $\left.\eta+\widetilde{B}_{t} \leq \widetilde{S}(t)\right\} \leq \widetilde{\tau}_{1}$, where $\widetilde{S}(t)=S\left(\rho^{-1}(t)\right)$ is increasing and $\widetilde{\tau}_{1}$ is the first hitting time to $S(0)$ of $B M$ $\widetilde{B}$ starting at $\eta$; since $\widetilde{\tau}_{1}$ is a.s. finite, also $\widetilde{\tau}$ is so. Next, from the finiteness of $\widetilde{\tau}$ it follows that $\tau=\rho^{-1}(\widetilde{\tau})$ is finite, too. Moreover, if one seeks that $E(\tau)<\infty$, a sufficient condition for this is that $\rho(t)$ and $\widetilde{S}(t)$ are both convex functions; indeed, $\widetilde{\tau} \leq \widetilde{\tau}_{2}$, where $\widetilde{\tau}_{2}$ is the FPT of BM $\widetilde{B}$ starting from $\eta$ below the straight line $a+b t\left(a=S(0) \geq 0, b=\widetilde{S}^{\prime}(0) \geq 0\right)$ which is tangent to the graph of $\widetilde{S}(t)$ at $t=0$. Thus, since $E\left(\widetilde{\tau}_{2}\right)<\infty$, it follows that $E(\widetilde{\tau})$ is finite, too; finally, being $\rho^{-1}$ concave, Jensen's inequality for concave functions implies that $E(\tau)=E\left(\rho^{-1}(\tilde{\tau})\right) \leq \rho^{-1}(E(\widetilde{\tau}))$ and therefore $E(\tau)<\infty$.

Remark 4. Theorem 1 allows to solve also the so called Skorokhod embedding (SE) problem:

Given a distribution $H$, find an integrable stopping time $\tau^{*}$, such that the distribution of $X\left(\tau^{*}\right)$ is $H$, namely $P\left(X\left(\tau^{*}\right) \leq x\right)=H(x)$.

In fact, let be $S(t)$ increasing, with $S(0)=0$; first suppose that the support of $H$ is $[0,+\infty)$; then, from Equation (4) it follows that

$$
\widehat{g}(\theta)=E\left[e^{-\theta X(\tau)-\frac{\theta^{2}}{2} \rho\left(S^{-1}(X(\tau))\right)}\right],
$$

and this solves the SE problem with $\tau^{*}=\tau$; it suffices to take the random initial point $X(0)=\eta>0$ in such a way that its Laplace transform $\widehat{g}$ satisfies

$$
\widehat{g}(\theta)=\int_{0}^{S(+\infty)} e^{-\theta x-\frac{\theta^{2}}{2} \rho\left(S^{-1}(x)\right)} d H(x) .
$$

In the special case when $S(t)=a+b t(a, b>0)$ and $\rho(t)=t$, Equation (7) becomes (cf. the result in [8] for $a=0)$ :

$$
\widehat{g}(\theta)=e^{\frac{a \theta^{2}}{2 b}} \widehat{h}\left(\frac{\theta(\theta+2 b)}{2 b}\right),
$$

where $h(x)=H^{\prime}(x)$ and $\widehat{h}$ denotes the Laplace transform of $h$.

In analogous way, the SE problem can be solved if the support of $H$ is $(-\infty, 0]$; now, the FPT is understood as $\tau^{-}=\inf \{t>0: \eta+B(\rho(t))>-S(t)\}(\eta<0)$, that is, the first hitting time to the boundary $S^{-}(t)=-S(t)$ from below.

Therefore, the solution to the general SE problem, namely without restrictions on the support of the distribution $H$, can be obtained as follows (see [8], for the case when $S(t)$ is a straight line).

The r.v. $X(\tau)$ can be represented as a mixture of the r.v. $X^{+}>0$ and $X^{-}<0$ :

$$
X(\tau)= \begin{cases}X^{+} & \text {with probability } p^{+}=P(X(\tau) \geq 0) \\ X^{-} & \text {with probability } p^{-}=1-p^{+}\end{cases}
$$

Suppose that the SE problem for the r.v. $X^{+}$and $X^{-}$can be solved by $S^{+}(t)=S(t)$ and $\eta^{+}=\eta>0$, and $S^{-}(t)=-S(t)$ and $\eta^{-}=-\eta<0$, respectively. Then, we get that the r.v.

$$
\eta^{ \pm}= \begin{cases}\eta^{+} & \text {with probability } p^{+} \\ \eta^{-} & \text {with probability } p^{-}\end{cases}
$$


and the boundary $S^{ \pm}(t)=S^{+}(t) \cup S^{-}(t)$ solve the SE problem for the r.v. $X(\tau)$.

If $\widehat{g}$ is analytic in a neighbor of $\theta=0$, then the moments of order $n$ of $\eta, E\left(\eta^{n}\right)$, exist finite, and they are given by $E\left(\eta^{n}\right)=\left.(-1)^{n} \frac{d^{n}}{d \theta^{n}} \widehat{g}\right|_{\theta=0}$. By taking the first derivative in Equation (4) and calculating it at $\theta=0$, we obtain

$$
E(\eta)=-\widehat{g}^{\prime}(0)=E(S(\tau)) .
$$

By calculating the second derivative of $\widehat{g}$ at $\theta=0$, we get

$$
\left.E\left(\eta^{2}\right)=\widehat{g}^{\prime \prime}(0)=E\left(S^{2}(\tau)-\rho(\tau)\right)\right),
$$

and so

$$
\operatorname{Var}(\eta)=E\left(\eta^{2}\right)-E^{2}(\eta)=\operatorname{Var}(S(\tau))-E(\rho(\tau)) .
$$

Thus, we obtain the compatibility conditions

$$
\left\{\begin{array}{l}
E(\eta)=E(S(\tau)) \\
\operatorname{Var}(S(\tau)) \geq E(\rho(\tau))
\end{array}\right.
$$

If $\operatorname{Var}(S(\tau))<E(\rho(\tau))$, a solution to the IFPT problem does not exist. In the special case when $S(t)=a+b t(a, b \geq 0)$ and $\rho(t)=t$, Equation (11) becomes $E(\eta)=a+b E(\tau)$ and Equation (13) becomes $\operatorname{Var}(\eta)=b^{2} \operatorname{Var}(\tau)-E(\tau)$, while Equation (14) coincides with Equation (2.3) of [2]. By writing the Taylor's expansions at $\theta=0$ of both members of Equation (4), and equaling the terms with the same order in $\theta$, one gets the successive derivatives of $\widehat{g}(\theta)$ at $\theta=0$; thus, one can write any moment of $\eta$ in terms of the expectation of a function of $\tau$; for instance, it is easy to see that

$$
\begin{gathered}
E\left(\eta^{3}\right)=E\left[(S(\tau))^{3}\right]-3 E[S(\tau) \rho(\tau)], \\
E\left(\eta^{4}\right)=E\left[\left(S(\tau)^{4}\right]-6 E\left[\left(S(\tau)^{2} \rho(\tau)\right]+3 E\left[\left(\rho(\tau)^{2}\right],\right.\right.\right. \\
E\left(\eta^{5}\right)=E\left[15 S(\tau) \rho^{2}(\tau)-240 S^{3}(\tau) \rho(\tau)+S^{5}(\tau)\right] .
\end{gathered}
$$

2.1. The Special Case $S(t)=\alpha+\beta \rho(t)$

If $S(t)=\alpha+\beta \rho(t)$, with $\alpha, \beta \geq 0$, from Equation (4) we get

$$
\widehat{g}(\theta)=E\left[e^{-\theta(\alpha+\beta \rho(\tau))-\frac{\theta^{2}}{2} \rho(\tau)}\right]=e^{-\theta \alpha} E\left[e^{-\theta \rho(\tau)(\beta+\theta / 2)}\right] .
$$

Thus, setting $\widetilde{\tau}=\rho(\tau)$, we obtain (see Equation (5)):

$$
\widehat{g}(\theta)=e^{-\theta \alpha} E\left[e^{-\theta(\beta+\theta / 2) \widetilde{\tau}}\right]=e^{-\theta \alpha} \widehat{\widetilde{f}}(\theta(\beta+\theta / 2)),
$$

having denoted by $\widetilde{f}$ the density of $\widetilde{\tau}$. In this way, we reduce the IFPT problem of $X(t)=\eta+B(\rho(t))$ below the boundary $S(t)=\alpha+\beta \rho(t)$ to that of BM below the linear boundary $\alpha+\beta t$. For instance, taking $\rho(t)=t^{3} / 3$, the solution to the IFPT problem of $X(t)$ through the cubic boundary $S(t)=\alpha+\frac{\beta}{3} t^{3}$, and the FPT density $f$, is nothing but the solution to the IFPT problem of BM through the linear boundary $\alpha+\beta t$, and the FPT density $\widetilde{f}$.

Under the assumption that $S(t)=\alpha+\beta \rho(t)$, with $\alpha, \beta \geq 0$, a number of explicit results can be obtained, by using the analogous ones which are valid for BM and a linear boundary (see [2]). As for the question of the existence of solutions to the IFPT problem, we have: 
Proposition 1. Let be $S(t)=\alpha+\beta \rho(t)$, with $\alpha, \beta \geq 0$; for $\gamma, \lambda>0$, suppose that the FPT density $f=F^{\prime}$ is given by

$$
f(t)= \begin{cases}\frac{\lambda^{\gamma}}{\Gamma(\gamma)} \rho(t)^{\gamma-1} e^{-\lambda \rho(t)} \rho^{\prime}(t) & \text { if } t>0 \\ 0 & \text { otherwise }\end{cases}
$$

(namely the density $\tilde{f}$ of $\widetilde{\tau}$ is the Gamma density with parameters $(\gamma, \lambda)$ ). Then, the IFPT problem has solution, provided that $\beta \geq \sqrt{2 \lambda}$, and the Laplace transform of the density $g$ of the initial position $\eta$ is given by:

$$
\widehat{g}(\theta)=\left[e^{-\alpha \theta / 2} \frac{\left(\beta-\sqrt{\beta^{2}-2 \lambda}\right)^{\gamma}}{\left(\theta+\beta-\sqrt{\beta^{2}-2 \lambda}\right)^{\gamma}}\right] \cdot\left[e^{-\alpha \theta / 2} \frac{\left(\beta+\sqrt{\beta^{2}-2 \lambda}\right)^{\gamma}}{\left(\theta+\beta+\sqrt{\beta^{2}-2 \lambda}\right)^{\gamma}}\right],
$$

which is the Laplace transform of the sum of two independent random variables, $Z_{1}$ and $Z_{2}$, such that $Z_{i}-\alpha / 2$ has distribution Gamma of parameters $\gamma$ and $\lambda_{i}(i=1,2)$, where $\lambda_{1}=\beta-\sqrt{\beta^{2}-2 \lambda}$ and $\lambda_{2}=\beta+$ $\sqrt{\beta^{2}-2 \lambda}$.

Remark 5. If $f$ is given by Equation (20), that is $\widetilde{f}$ is the Gamma density, the compatibility condition in Equation (14) becomes $\beta \geq \sqrt{\lambda}$, which is satisfied under the assumption $\beta \geq \sqrt{2 \lambda}$ required by Proposition 1 . In the special case when $\gamma=1$, then $\eta$ has the same distribution as $\alpha+Z_{1}+Z_{2}$, where $Z_{i}$ are independent and exponential with parameter $\lambda_{i}, i=1,2$.

The following result also follows from Proposition 2.5 of [2].

Proposition 2. Let be $S(t)=\alpha+\beta \rho(t)$, with $\alpha, \beta \geq 0$; for $\beta>0$, suppose that the Laplace transform of $\tilde{f}$ has the form:

$$
\widehat{\widetilde{f}}(\theta)=\sum_{k=1}^{N} \frac{A_{k}}{\left(\theta+B_{k}\right)^{c_{k}}}
$$

for some $c_{k}>0, A_{k}, B_{k}>0, k=1, \ldots, N$. Then, there exists a value $\beta^{*}>0$ such that the solution to the IFPT problem exists, provided that $\beta \geq b^{*}$.

If $\beta=0$ and the Laplace transform of $\tilde{f}$ has the form:

$$
\widehat{\widetilde{f}}(\theta)=\sum_{k=1}^{N} \frac{A_{k}}{\left(\sqrt{2 \theta}+B_{k}\right)^{c_{k}}}
$$

then, the solution to the IFPT problem exists.

\subsection{Approximate Solution to the IFPT Problem for Non Linear Boundaries}

Now, we suppose that there exist $\alpha_{1}, \alpha_{2}, \beta_{1}, \beta_{2}$ with $0 \leq \alpha_{1} \leq \alpha_{2}$ and $\beta_{2} \geq \beta_{1} \geq 0$, such that, for every $t \geq 0$ :

$$
\alpha_{1}+\beta_{1} \rho(t) \leq S(t) \leq \alpha_{2}+\beta_{2} \rho(t)
$$

namely $S(t)$ is enveloped from above and below by the functions $S_{\alpha_{2}, \beta_{2}}(t)=\alpha_{2}+\beta_{2} \rho(t)$ and $S_{\alpha_{1}, \beta_{1}}(t)=\alpha_{1}+\beta_{1} \rho(t)$.

Then, by using Proposition (3.13) of [16] (see also [1]), we obtain the following:

Proposition 3. Let $S(t)$ a continuous, increasing boundary satisfying Equation (24) and suppose that the FPT $\tau$ of $X(t)=\eta+B(\rho(t))(\eta>S(0))$ below the boundary $S(t)$ has an assigned probability density $f$ and that there exists a density $g$ with support $(S(0),+\infty)$, which is solution to the IFPT problem for $X(t)$ and the boundary $S(t)$; as before, denote by $\widetilde{f}(t)$ the density of $\rho(\tau)$ and by $\widetilde{\widetilde{f}}(\theta)$ its Laplace transform, for $\theta>0$. Then: 
(i) If $\alpha_{2}>\alpha_{1}$ and the function $g \in L^{p}\left(S(0), \alpha_{2}\right)$ for some $p>1$, its Laplace transform $\widehat{g}(\theta)$ must satisfy:

$$
\begin{gathered}
e^{-\alpha_{2}\left(\theta+2\left(\beta_{2}-\beta_{1}\right)\right)[}\left[\widehat{\widetilde{f}}\left(\frac{\theta\left(\theta+2 \beta_{2}\right)}{2}\right)-\left(\alpha_{2}-S(0)\right)^{\frac{p-1}{p}}\left(\int_{S(0)}^{\alpha_{2}} g^{p}(x) d x\right)^{1 / p}\right] \leq \widehat{g}(\theta) \\
\leq e^{-\alpha_{1} \theta} \widehat{\widetilde{f}}\left(\frac{\theta\left(\theta+2 \beta_{1}\right)}{2}\right) ;
\end{gathered}
$$

(ii) If $\alpha_{1}=\alpha_{2}=S(0)$, then Equation (25) holds without any further assumption on $g$ (and the term $\left(\alpha_{2}-S(0)\right)^{\frac{p-1}{p}}\left(\int_{S(0)}^{\alpha_{2}} g^{p}(x) d x\right)^{1 / p}$ vanishes $)$.

Remark 6. The smaller $\alpha_{2}-\alpha_{1}$ and $\beta_{2}-\beta_{1}$, the better the approximation to the Laplace transform of $g$. Notice that, if $g$ is bounded, then the term $\left(\alpha_{2}-S(0)\right)^{\frac{p-1}{p}}\left(\int_{S(0)}^{\alpha_{2}} g^{p}(x) d x\right)^{1 / p}$ can be replaced with $\left(\alpha_{2}-S(0)\right)\|g\|_{\infty}$.

\subsection{The IFPT Problem for $\bar{X}(t)=\eta+B(\rho(t))+$ Large Jumps}

As an application of the previous results, we consider now the piecewise-continuous process $\bar{X}(t)$, obtained by superimposing to $X(t)$ a jump process, namely we set $\bar{X}(t)=\eta+B(\rho(t))$ for $t<T$, where $T$ is an exponential distributed time with parameter $\mu>0$; we suppose that, for $t=T$ the process $\bar{X}(t)$ makes a downward jump and it crosses the continuous increasing boundary $S$, irrespective of its state before the occurrence of the jump. This kind of behavior is observed e.g. in the presence of a so called catastrophes (see e.g., [17]). For $\eta \geq S(0)$, we denote by $\bar{\tau}_{S}=\inf \{t>0: \bar{X}(t) \leq S(t)\}$ the FPT of $\bar{X}(t)$ below the boundary $S(t)$. The following holds:

Proposition 4. If there exists a solution $\bar{g}$ to the IFPT problem of $\bar{X}(t)$ below $S(t)$ with $\bar{X}(0)=\eta \geq S(0)$, then its Laplace transform is given by

$$
\widehat{\bar{g}}(\theta)=E\left[e^{-\theta S(\tau)-\frac{\theta^{2}}{2} \rho(\tau)-\mu \tau}\right]+\mu \int_{0}^{+\infty} e^{-\theta S(t)-\frac{\theta^{2}}{2} \rho(t)-\mu t}\left(\int_{t}^{+\infty} f(s) d s\right) d t .
$$

Proof. For $t>0$, one has:

$$
P\left(\bar{\tau}_{S} \leq t\right)=P\left(\bar{\tau}_{S} \leq t \mid t<T\right) P(t<T)+1 \cdot P(t \geq T)=P\left(\tau_{S} \leq t\right) e^{-\mu t}+\left(1-e^{-\mu t}\right)
$$

Taking the derivative, one obtains the FPT density of $\bar{\tau}$ :

$$
\bar{f}(t)=e^{-\mu t} f(t)+\mu e^{-\mu t} \int_{t}^{+\infty} f(s) d s
$$

where $f$ is the density of $\tau$. Then, by the same arguments used in the proof of Theorem 1 , we obtain

$$
\begin{gathered}
\widehat{\bar{g}}(\theta)=E\left[e^{-\theta S(\bar{\tau})-\frac{\theta^{2}}{2} \rho(\bar{\tau})}\right] \\
=\int_{0}^{\infty} e^{-\theta S(t)-\frac{\theta^{2}}{2} \rho(t) \bar{f}(t) d t} \\
=\int_{0}^{\infty} e^{-\theta S(t)-\frac{\theta^{2}}{2} \rho(t)}\left[e^{-\mu t} f(t)+\mu e^{-\mu t} \int_{t}^{\infty} f(s) d s\right] d t \\
=\int_{0}^{\infty} e^{-\theta S(t)-\frac{\theta^{2}}{2} \rho(t)-\mu t} f(t) d t+\mu \int_{0}^{\infty} e^{-\theta S(t)-\frac{\theta^{2}}{2} \rho(t)-\mu t}\left(\int_{t}^{\infty} f(s) d s\right) d t
\end{gathered}
$$

that is Equation (26).

Remark 7. (i) For $\mu=0$, namely when no jump occurs, Equation (26) becomes Equation (4). 
(ii) If $\tau$ is exponentially distributed with parameter $\lambda$, then Equation (26) provides:

$$
\widehat{\bar{g}}(\theta)=\frac{\lambda+\mu}{\lambda} E\left[e^{-\theta S(\tau)-\frac{\theta^{2}}{2} \rho(\tau)-\mu \tau}\right] .
$$

(iii) In the special case when $S(t)=\alpha+\beta \rho(t)(\alpha, \beta \geq 0)$, we can reduce to the FPT $\overline{\widetilde{\tau}}$ of BM + large jumps below the linear boundary $\alpha+\beta$ t; then, it is possible to write $\widehat{\bar{g}}$ in terms of the Laplace transform of $\overline{\widetilde{\tau}}$. Really, by using Proposition 3.10 of [16] one gets

$$
\widehat{\bar{g}}(\theta)=e^{-\alpha \theta}\left[\left(1-\frac{2 \mu}{\theta(\theta+2 \beta)}\right)^{-1} \widehat{\bar{f}}\left(\frac{\theta(\theta+2 \beta)}{2}-\mu\right)-\frac{2 \mu}{\theta(\theta+2 \beta)-2 \mu}\right],
$$

where, for simplicity of notation we have denoted again with $\widehat{\bar{f}}$ the Laplace transform of $\overline{\tilde{\tau}}$; of course, if $\rho(t)=t$, then $\widehat{\bar{f}}$ is the Laplace transform of $\bar{\tau}$. Notice that, if $\mu=0$ the last equation is nothing but Equation (5) with $\alpha, \beta$ in place of $a, b$.

\section{Some Examples}

Example 1. If $S(t)=a+b t$, with $a, b \geq 0$, and $X(t)=B_{t}(\rho(t)=1)$, examples of solution to the IFPT problem, for $X(t)$ and various FPT densities $f$, can be found in [2].

Example 2. Let be $S(t)=\alpha+\beta \rho(t)$, with $\alpha, \beta \geq 0$, and suppose that $\tau$ has density $f(t)=$ $\lambda e^{-\rho(t)} \rho^{\prime}(t) \mathbf{1}_{(0,+\infty)}(t)$ (that is, the density $\tilde{f}$ of $\widetilde{\tau}=\rho(\tau)$ is exponential with parameter $\lambda$ ). By using Proposition 1 we get that $\eta=\alpha+Z_{1}+Z_{2}$, where $Z_{i}$ are independent random variable, such that $Z_{i}-\alpha / 2$ has exponential distribution with parameter $\lambda_{i}(i=1,2)$, where $\lambda_{1}=\beta-\sqrt{\beta^{2}-2 \lambda}$ and $\lambda_{2}=\beta+\sqrt{\beta^{2}-2 \lambda}$. Then, the solution $g$ to the IFPT problem for $X(t)=\eta+B(\rho(t))$, the boundary $S$ and the exponential FPT distribution, is:

$$
g(x)=\left\{\begin{array}{l}
\frac{\lambda_{1} \lambda_{2}}{\lambda_{2}-\lambda_{1}} e^{-\lambda_{1}(x-\alpha)}-e^{-\lambda_{2}(x-\alpha)}, \text { if } b>\sqrt{2 \lambda} \\
2 \lambda(x-\alpha) e^{-\sqrt{2 \lambda}(x-a)}, \text { if } b=\sqrt{2 \lambda}
\end{array} \quad(x \geq \alpha)\right.
$$

In general, for a given continuous increasing boundary $S(t)$ and an assigned distribution of $\tau$, it is difficult to calculate explicitly the expectation on the right-hand member of Equation (4) to get the Laplace transform of $\eta$. Thus, a heuristic solution to the IFPT problem can be achieved by using Equation (4) to calculate the moments of $\eta$ (those up to the fifth order are given by Equations (11), (12) and (15)-(17)). Of course, even if one was able to find the moments of $\eta$ of any order, this would not determinate the distribution of $\eta$. However, this procedure is useful to study the properties of the distribution of $\eta$, provided that the solution to the IFPT problem exists.

Example 3. Let be $S(t)=t^{2}, \rho(t)=t$ and suppose that $\tau$ is exponentially distributed with parameter $\lambda$; we search for a solution $\eta>0$ to the IFPT problem by using the method of moments, described above. The compatibility condition in Equation (14) requires that $\lambda^{3}<20$ (for instance, one can take $\lambda=1$ ). From Equations (11), (12) and (15)-(17), and calculating the moments of $\tau$ up to the eighth order, we obtain:

$$
\begin{gathered}
E(\eta)=E\left(\tau^{2}\right)=\frac{2}{\lambda^{2}} ; E\left(\eta^{2}\right)=E\left(\tau^{4}\right)-E(\tau)=\frac{24-\lambda^{3}}{\lambda^{4}} ; \sigma^{2}(\eta)=\operatorname{Var}(\eta)=\frac{20-\lambda^{3}}{\lambda^{4}} \\
E\left(\eta^{3}\right)=E\left(\tau^{6}\right)-3 E\left(\tau^{3}\right)=\frac{720-18 \lambda^{3}}{\lambda^{6}} ; E\left(\eta^{4}\right)=E\left(\tau^{8}\right)-6 E\left(\tau^{3}\right)+3 E\left(\tau^{2}\right)=\frac{8 !-36 \lambda^{5}+6 \lambda^{6}}{\lambda^{8}} .
\end{gathered}
$$

Notice that, under the condition $\lambda^{3}<20$ the first four moments of $\eta$ are positive, as it must be. However, they do not match those of a Gamma distribution.

An information about the asymmetry is given by the skewness value

$$
\frac{E(\eta-E(\eta))^{3}}{\sigma(\eta)^{3}}=-12 \frac{24-\lambda^{3}}{\left(20-\lambda^{3}\right)^{3 / 2}}<0
$$


meaning that the candidate $\eta$ has an asymmetric distribution with a tail toward the left.

\section{Conclusions and Final Remarks}

We have dealt with the IFPT problem for a continuously time-changed Brownian motion $X(t)$ starting from a random position $\eta$. For a given continuous, increasing boundary $S(t)$ with $\eta \geq S(0) \geq$ 0 , and an assigned continuous distribution function $F$, the IFPT problem consists in finding the distribution, or the density $g$ of $\eta$, such that the first-passage time $\tau$ of $X(t)$ below $S(t)$ has distribution $F$. In this note, we have provided some extensions of the results, already known in the case when $X(t)$ is $\mathrm{BM}$ and $S(t)$ is a straight line, and we have reported some explicit examples. Really, the process we considered has the form $X(t)=\eta+\int_{0}^{t} \sigma(s) d B_{s}$, where $B_{t}$ is standard Brownian motion, and $\sigma(t)$ is a non random continuous function of time $t \geq 0$, such that the function $\rho(t)=\int_{0}^{t} \sigma^{2}(s) d s$ is increasing and it satisfies the condition $\rho(+\infty)=+\infty$. Thus, a standard BM $\widehat{B}$ exists such that $X(t)=\eta+\widehat{B}(\rho(t))$. Our main result states that

$$
\widehat{g}(\theta)=E\left[e^{-\theta S(\tau)-\frac{\theta^{2}}{2} \rho(\tau)}\right],
$$

where, for $\theta>0, \widehat{g}(\theta)$ denotes the Laplace transform of the solution $g$ to the IFPT problem.

Notice that the above result can be extended to diffusions which are more general than the process $X(t)$ considered, for instance to a process of the form

$$
U(t)=w^{-1}(\widehat{B}(\rho(t))+w(\eta)),
$$

where $w$ is a regular enough, increasing function; such a process $U$ is obtained from BM by a space transformation and a continuous time-change (see e.g., the discussion in [2]). Since $w(U(t))=$ $w(\eta)+\widehat{B}(\rho(t))$, the IFPT problem for the process $U$, the boundary $S(t)$ and the FPT distribution $F$, is reduced to the analogous IFPT problem for $X(t)=\eta_{1}+\widehat{B}(\rho(t))$, starting from $\eta_{1}=w(\eta)$, instead of $\eta$, the boundary $S_{1}(t)=w(S(t))$ and the same FPT distribution $F$. When $\sigma(t)=1$, i.e. $\rho(t)=t$, the process $U(t)$ is conjugated to BM, according to the definition given in [2]; two examples of diffusions conjugated to BM are the Feller process, and the Wright-Fisher like (or CIR) process, (see e.g., [2]). The process $U(t)$ given by Equation (32) is indeed a weak solution of the SDE:

$$
d U(t)=-\frac{\rho^{\prime}(t) w^{\prime \prime}(U(t))}{2\left(w^{\prime}(U(t))\right)^{3}} d t+\frac{\sqrt{\rho^{\prime}(t)}}{w^{\prime}(U(t))} d B_{t},
$$

where $w^{\prime}(x)$ and $w^{\prime \prime}(x)$ denote first and second derivative of $w(x)$.

Provided that the deterministic function $\rho(t)$ is replaced with a random function, the representation in Equation (32) is valid also for a time homogeneous one-dimensional diffusion driven by the SDE

$$
d U(t)=\mu(U(t)) d t+\sigma(U(t)) d B_{t}, U(0)=\eta,
$$

where the drift $(\mu)$ and diffusion coefficients $(\sigma)$ satisfy the usual conditions (see e.g., [18]) for existence and uniqueness of the solution of Equation (34). In fact, let $w(x)$ be the scale function associated to the diffusion $U(t)$ driven by the SDE Equation (34), that is, the solution of $L w(x)=0, w(0)=0, w^{\prime}(0)=1$, where $L$ is the infinitesimal generator of $U$ given by $L h=\frac{1}{2} \sigma^{2}(x) \frac{d^{2} h}{d x^{2}}+\mu(x) \frac{d h}{d x}$. As easily seen, if the integral $\int_{0}^{t} \frac{2 \mu(z)}{\sigma^{2}(z)} d z$ converges, the scale function is explicitly given by

$$
w(x)=\int_{0}^{x} \exp \left(-\int_{0}^{t} \frac{2 \mu(z)}{\sigma^{2}(z)} d z\right) d t .
$$

If $\zeta(t):=w(U(t))$, by Itô's formula one obtains

$$
\zeta(t)=w(\eta)+\int_{0}^{t} w^{\prime}\left(w^{-1}(\zeta(s))\right) \sigma\left(w^{-1}(\zeta(s))\right) d B_{s},
$$


that is, the process $\zeta(t)$ is a local martingale, whose quadratic variation is

$$
\rho(t) \doteq\langle\zeta\rangle_{t}=\int_{0}^{t}\left[w^{\prime}(U(s)) \sigma(U(s))\right]^{2} d s, t \geq 0
$$

The (random) function $\rho(t)$ is differentiable and $\rho(0)=0$; if it is increasing to $\rho(+\infty)=+\infty$, by the Dambis, Dubins-Schwarz theorem (see e.g., [3]) one gets that there exists a standard BM $\widehat{B}$ such that $\zeta(t)=\widehat{B}(\rho(t))+w(\eta)$. Thus, since $w$ is invertible, one obtains the representation in Equation (32).

Notice, however, that the IFPT problem for the process $U$ given by Equation (32) cannot be addressed as in the case when $\rho$ is a deterministic function. In fact, if $\rho(t)$ given by Equation (37) is random, it results that $\rho(t)$ and the FPT $\tau$ are dependent. Thus, in line of principle it would be possible to obtain information about the Laplace transform of $g$, only in the case when the joint distribution of $(\rho(t), \tau)$ was explicitly known.

Funding: This research was funded by the MIUR Excellence Department Project awarded tothe Department of Mathematics, University of Rome Tor Vergata, CUP E83C18000100006.

Acknowledgments: I would like to express particular thanks to the anonymous referees for their constructive comments and suggestions leading to improvements of the paper.

Conflicts of Interest: The author declares no conflict of interest.

\section{References}

1. Abundo, M. Some randomized first-passage problems for one-dimensional diffusion processes. Sci. Math. Jpn. 2013, 76, 33-46.

2. Abundo, M. An inverse first-passage problem for one-dimensional diffusion with random starting point. Stat. Probab. Lett. 2012, 82, 7-14. [CrossRef]

3. Revuz, D.; Yor, M. Continous Martingales and Brownian Motion; Springer: Berlin/Heidelberg, Germany, 1991.

4. Darling, D.A.; Siegert, A.J.F. The first passage problem for a continuous Markov process. Ann. Math. Stat. 1953, 24, 264-639. [CrossRef]

5. Hieber, P.; Scherer, M. A note on first-passage times of continuously time-changed Brownian motion. Stat. Probab. Lett. 2012, 82, 165-172. [CrossRef]

6. Hurd, T.R. Credit risk modeling using time-changed Brownian motion. Int. J. Theor. Appl. Financ. 2009, 12, 1213-1230. [CrossRef]

7. Jackson, K.; Kreinin, A.; Zhang, W. Randomization in the first hitting problem. Stat. Probab. Lett. 2009, 79, 2422-2428. [CrossRef]

8. Jaimungal, S.; Kreinin, A.; Valov, A. The generalized Shiryaev problem and Skorokhod embedding. Theory Probab. Appl. 2014, 58, 493-502. [CrossRef]

9. Lanska, V.; Smiths, C.E. The effect of a random initial value in neural first-passage-time models. Math. Biosci. 1989, 93, 191-215. [CrossRef]

10. Peskir, G. On integral equations arising in the first-passage problem for Brownian motion. J. Integral Equat. Appl. 2002, 14, 397-423. [CrossRef]

11. Sacerdote, L.; Zucca, C. Threshold shape corresponding to a Gamma firing distribution in an Ornstein-Uhlenbeck neuronal model. Sci. Math. Jpn. 2003, 19, 1319-1346.

12. Zucca, C.; Sacerdote, L. On the inverse first-passage-time problem for a Wiener process. Ann. Appl. Probab. 2009, 8, 375-385. [CrossRef]

13. Abundo, M. Limit at zero of the first-passage time density and the inverse problem for one-dimensional diffusions. Stoch. Anal. Appl. 2006, 24, 1119-1145. [CrossRef]

14. Klebaner, F.C. Introduction to Stochastic Calculus With Applications; Imperial College Press: London, UK, 2005.

15. Di Nardo, E.; Nobile, A.G.; Pirozzi, E.; Ricciardi, L.M. A computational approach to first-passage-time problems for Gauss-Markov processes. Adv. Appl. Probab. 2001, 33, 453-482. [CrossRef]

16. Abundo, M. An overview on inverse first-passage-time problems for one-dimensional diffusion processes. Lect. Notes Semin. Interdiscip. Matematica 2015, 12,1-44. Available online: http:/ /dimie.unibas.it/site/home/ info/documento3012448.html (accessed on 28 May 2018) 
17. Di Crescenzo, A.; Giorno, V.; Nobile, A.G.; Ricciardi, L.M. On the M/M/1 queue with catastrophes and its continuous approximation. Queueing Syst. 2003, 43, 329-347. [CrossRef]

18. Ikeda, N.; Watanabe, S. Stochastic Differential Equations and Diffusion Processes; North-Holland Publishing Company: Amsterdam, The Netherlands, 1981.

(C) 2018 by the author. Licensee MDPI, Basel, Switzerland. This article is an open access article distributed under the terms and conditions of the Creative Commons Attribution (CC BY) license (http:/ / creativecommons.org/licenses/by/4.0/). 